\title{
Geometrically asymmetric electrodes for probing electrochemical reaction kinetics: a case study of hydrogen at the $\mathrm{Pt}-\mathrm{CsH}_{2} \mathrm{PO}_{4}$ interface
}

\author{
Kenji A. Sasaki, Yong Hao and Sossina M. Haile* \\ Received 13th May 2009, Accepted 9th June 2009 \\ First published as an Advance Article on the web 15th July 2009 \\ DOI: $10.1039 / b 909498 a$
}

Electrochemical reactions can exhibit considerable asymmetry, with the polarization behavior of oxidation at a given metal|electrolyte interface differing substantially from that of reduction. The reference-less, microcontact electrode geometry, in which the electrode overpotentials are geometrically constrained to the working electrode (by limiting its area) is experimentally convenient, particularly for fuel cell studies, because the results do not rely on accurate placement of a reference electrode nor must oxidant and reductant gases be sealed off from one another. Here, the conditions under which the critical assumption of this geometry applies - that the overpotential at the large-area counter electrode can be ignored - is numerically assessed. It is found that, for cells of sufficiently large area, the effective radius of the counter electrode (which defines the area through which the majority of the current passes) can be expressed directly as a function of electrolyte thickness and the materials properties, $\sigma$, the conductivity of the electrolyte, and $k$, the reaction rate constant for the electrochemical reaction at zero-bias. From this effective radius and the true radius of the working electrode, the fraction of electrode overpotential at the latter, defined as the extent of isolation, can be readily computed. Experimental studies of hydrogen electro-oxidation/proton electro-reduction at the $\mathrm{Pt} \mid \mathrm{CsH}_{2} \mathrm{PO}_{4}$ interface using two cells of differing dimensions both validate the computational results and demonstrate that asymmetry in such reactions are readily revealed in the micro-electrode, reference-less geometry. The study furthermore confirms the insensitivity of the results to the precise placement of the working electrode, while indicating the importance of very high isolation values $(>99 \%)$ to ensure that overpotential contributions of the counter electrode do not influence the measurements, particularly as bias is increased.

\section{Introduction}

The quest for high power output fuel cells can be largely equated to the quest for high performance components (anode, cathode, electrolyte) which induce as small a voltage drop as possible for a given fuel cell current. The currentvoltage (or $I-V$ ) characteristics of common electrolyte materials generally obey Ohm's law and as such minimization of the voltage drop across the electrolyte is a straight-forward matter of either increasing the ionic conductivity or decreasing the electrolyte thickness (or a combination thereof). In contrast, the $I-V$ characteristics of fuel cell electrodes can vary widely, and careful measurements are required if one is to elucidate electrochemical reaction pathways and ultimately create high performance fuel cells.

A relatively common method for the study of electrode characteristics is alternating current impedance spectroscopy at zero bias in a symmetric gas environment. That is, nominally identical gas/electrode/electrolyte interfaces are present on both sides of the cell, and, from the a.c. response, one obtains the effective interfacial impedance. In such a configuration, any forward reaction at one electrode is balanced by the

Materials Science, California Institute of Technology, Pasadena,

CA91125,USA.E-mail:smhaile@caltech.edu reverse reaction at the second electrode. As a consequence, it is not possible from such a measurement to separately probe the forward and reverse reactions, for example, of hydrogen electro-oxidation/proton electro-reduction

$$
H_{2} \underset{\text { reverse }}{\stackrel{\text { forward }}{\rightleftarrows}} 2 H^{+}+2 e^{-}
$$

Thus, the true $I-V$ characteristics of the electrochemical reaction of interest cannot be determined by this method.

The use of a reference electrode, placed at an appropriate location, can circumvent the limitations of a two-electrode geometry and reveal, independently, the behavior of the two reactions implied by eqn (1). This strategy is often implemented in conjunction with an asymmetric gaseous environment in which one electrode is supplied with the reductant gas and the second with the oxidant-creating a voltage across the electrolyte - much as in fuel cell operation. While this approach has provided important insight in many instances, the challenges inherent in properly placing the reference electrode and in obtaining sufficient seals are non-trivial and can generate results that are difficult to interpret. ${ }^{1,2}$

An alternative to the reference-electrode method for the study of asymmetry in electrochemical reactions, specifically, reactions on solid oxygen ion conductors, was proposed by 
Fabry and Kleitz in $1974 .^{3}$ These authors outlined a geometric approach in which the interfacial area of the working electrode is made very small relative to that of the counter electrode. It is then presumed that, as a consequence of this geometric asymmetry, the observed electrode response is dominated by the behavior of the working electrode. Thus, at non-zero current flow, with the reaction at the working electrode occurring in only one direction, the polarization characteristics specific to this direction are revealed. The asymmetric geometry does not eliminate the contribution of the electrolyte to the total voltage drop of the electrochemical cell; however, the electrolyte characteristics can be evaluated using either current interrupt methods or impedance spectroscopy.

In recent years, geometrically well-defined point and patterned microelectrodes have received increasing attention from the fuel cell community. However, few researchers have exploited the benefits of the resultant constriction of the electrochemical current at the working electrode. In most instances a reference electrode is retained as part of the configuration. ${ }^{4-9}$ In contrast to this trend, Fleig and co-workers ${ }^{10-14}$ have adopted a point electrode geometry that is explicitly reference-less. For dense, surface-limited electrodes, the assumption that the point geometry has effectively eliminated the contribution of the counter electrode is likely valid. However, the microstructure examined bears little relation to composite electrocatalyst mixtures employed in 'real' fuel cells. Indeed, our preliminary studies using $\mathrm{Pt}$ indicate a substantial difference in the electrochemical behavior between solid and porous electrodes. ${ }^{15}$ In 2008, Uda et al. proposed a refinement of the reference-less method $^{16}$ in which the area of the working electrode is restricted, but the microstructure of interest is retained. In principle, this allows one to take advantage of a measurement geometry that is free of complications due to misalignment of reference electrodes and apply it to a 'realistic' composite electrode. Again, however, it is assumed, without direct validation, that the response of the counter electrode is negligible.

In the present work we carry out a combined computational and experimental study to determine under which conditions the critical assumption of the reference-less geometry-that the counter electrode can be neglected-applies. The model is verified, and the impact of the counter-electrode is discussed in the specific context of electrochemical reactions at the interface between platinum and the solid acid electrolyte $\mathrm{CsH}_{2} \mathrm{PO}_{4}$. Viability of this electrolyte for fuel cell applications has been demonstrated ${ }^{17}$ and, furthermore, for cells fabricated with $25 \mu \mathrm{m}$ thick membranes, it has been shown that electrocatalysis is rate-limiting. ${ }^{18}$ Hence, study of the $\mathrm{Pt}-\mathrm{CsH}_{2} \mathrm{PO}_{4}$ interface has both scientific and technological relevance. In the specific implementation of the reference-less geometry here, the electrolyte contribution to the system voltage drop is experimentally determined by a.c. impedance spectroscopy. While this technique provides the added advantage of revealing the occurrence of any multi-step electrode processes, a detailed examination of electrochemical reaction pathways is beyond the scope of the present study.

\section{Model description}

The geometry of interest, which displays cylindrical symmetry, is shown in Fig. 1. An electrolyte disc of thickness $t$ and radius $r_{0}$ is contacted along the entirety of one of its faces with a counter electrode and on the opposing face with a centrally placed microelectrode of radius $r_{\mathrm{WE}}$. The voltage at the counter electrode is set at zero, and a fixed nonzero voltage is applied at the working electrode.

The resulting potential distribution within the electrochemical cell, $\phi(r, y)$ is a function of both radial position $r$ and vertical position $y$ and is governed by Laplace's equation, given in cylindrical coordinates as

$$
\frac{1}{r} \frac{\partial}{\partial r}\left(r \frac{\partial \phi}{\partial r}\right)+\frac{\partial^{2} \phi}{\partial y^{2}}=0
$$

The (ionic) current density, $j$, through the electrolyte depends on the potential gradient according to Ohm's law, which, for a cylindrical coordinate system and an isotropic medium is given as

$$
\begin{aligned}
& j=-\sigma \nabla \phi(r, y)=j_{r}(r, y) \hat{\boldsymbol{r}}+j_{y}(r, y) \hat{\boldsymbol{y}} \\
& j_{r}=-\sigma \frac{\partial \phi}{\partial r}, j_{y}=-\sigma \frac{\partial \phi}{\partial y}
\end{aligned}
$$

where $\sigma$ is the ionic conductivity of the electrolyte (taken to be isotropic and independent of position), $j_{r}$ and $j_{y}$ are the current densities in the radial and vertical directions, respectively, and $\hat{\boldsymbol{r}}$ and $\hat{\boldsymbol{y}}$ are the unit vectors in the $r$ and $y$ directions, respectively.

At the electrodes, the finite electrocatalysis kinetics generate a potential drop across each of the electrode/electrolyte interfaces, beyond the potential change induced by electrochemical equilibration between the metal and electrolyte. This potential drop, referred to as the overpotential, $\eta$, in the general case, displays a non-linear dependence on current, as already noted above. At sufficiently small overpotentials, however, the behavior can be approximated as linear, and the interfacial current density, $j_{\perp}$, is proportional to the overpotential according to

$$
j_{\perp}=k \eta= \pm k\left(\phi^{\text {electrolyte }}-\phi_{0}^{\text {metal }}\right)
$$

where the proportionality constant, $k$, is the reaction rate constant (inversely proportional to the interfacial area specific resistance and also taken to be independent of position), $\phi^{\text {electrolyte }}$ is the potential at the electrolyte side of the interface, and $\phi_{0}^{\text {metal }}$ is the potential at the metal side of the interface, and, where appropriate, additional subscripts WE and CE are used to specify these terms as being those of the working or counter electrode, respectively. (The specification \pm ensures that the overpotentials at the two electrodes are of the same sign, with + for the working electrode and - for the counter electrode.) For computational convenience but without loss of generality, in writing eqn (5) the equilibrium Galvani potential change at the electrode-electrolyte interface is taken to be zero. This boundary condition further relates the overpotential 

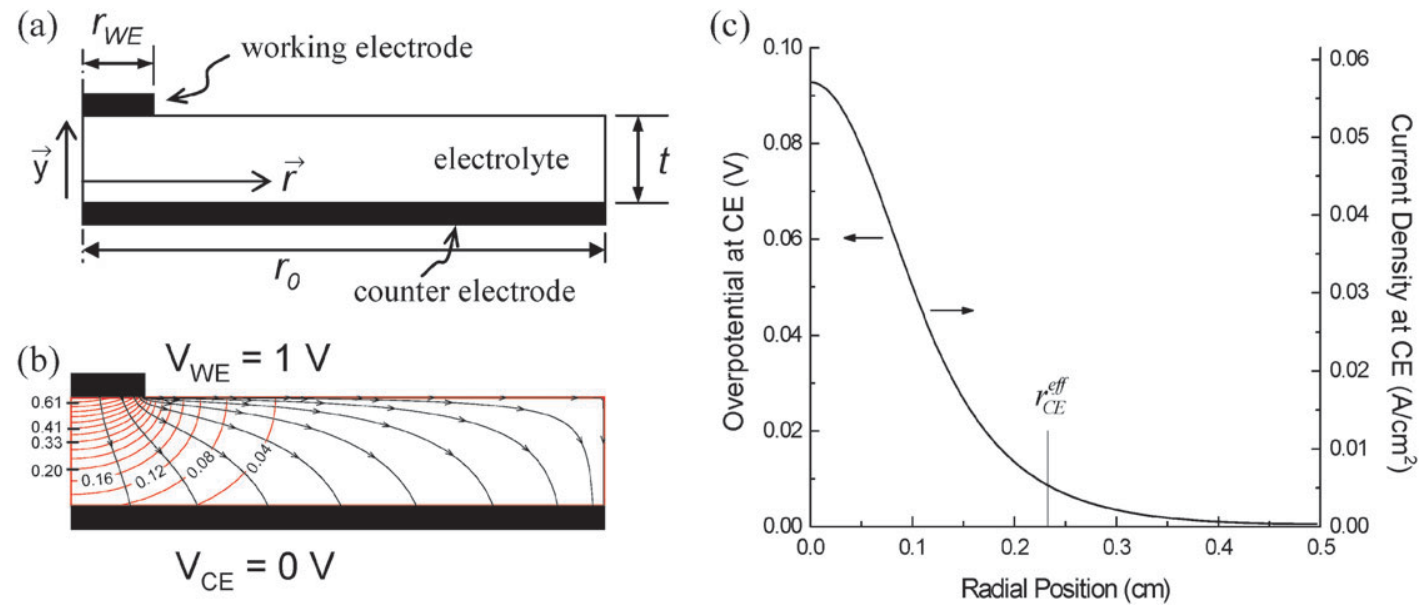

Fig. 1 (a) Cell geometry in the reference-less configuration; (b) example of calculated equipotential and current vector lines (with selected potential values as indicated); and (c) of overpotential and current density at the interface between the electrolyte and the counter electrode. Calculations performed for $k=0.615 \Omega^{-1} \mathrm{~cm}^{-2}, \sigma=0.021 \Omega^{-1} \mathrm{~cm}^{-1}, t=0.1 \mathrm{~cm}, r_{\mathrm{WE}}=0.0691 \mathrm{~cm}$, and $r_{0}=0.5 \mathrm{~cm}$.

at the electrode to the electrolyte properties. Specifically, considering eqn (4) and (5) one obtains

$$
\eta=\frac{j_{\perp}}{k}=\left.\frac{-\sigma}{k} \frac{\partial \phi}{\partial \hat{y}}\right|_{y=0, t}
$$

The boundary conditions at the remaining electrolyte surfaces, including the exposed upper boundary (i.e. that not covered by the working electrode) and the two vertical boundaries at $r=0$ and $r=r_{0}$ are simply that the flux is equal to zero. At $r=0$ the zero flux condition results from the axial symmetry of the electrochemical cell. At the other two boundaries, the zero flux condition is a result of electrical insulation.

Along with the transport and Laplace equations, the boundary conditions and the material properties, $\sigma$ and $k$, fully define the potential profile within the electrochemical cell. The solution, $\phi(r, y)$, is obtained here numerically using an in-house developed program based on the finite-volume formalism and finite-difference discretization. ${ }^{19}$ The finite volume approach enables representation of partial differential equations as algebraic equations, whereas the finite difference discretization provides the specific algebraic expression. Here, Laplace's eqn (2) is discretized on an axisymmetric cylindrical coordinate system within the electrolyte. Since both eqn (2) and the boundary conditions (5) are linear, the discretization yields a system of linear algebraic equations with the unknowns being the values $\left\{\phi_{i}\right\}$ defined at each grid point. This system of equations is then solved iteratively to yield $\left\{\phi_{i}\right\}$. Because the gradient in the potential is much greater in the vicinity of the working electrode than in that of the counter electrode, the numerical mesh used for the computation was made fine in the vicinity of the electrode interfaces (in the regions close to $r=0, r=r_{\mathrm{WE}}$ and $y=0, y=t$ ) and progressively coarsened towards the center and insulating boundaries. For a given mesh, convergence of $\left\{\phi_{i}\right\}$ was defined to be satisfied when the relative variation of each $\phi_{i}$ became smaller than a specified value, typically $10^{-8}$, whereas the appropriate mesh resolution was assured by computing $\left\{\phi_{i}\right\}$ for progressively finer meshes until the results became insensitive to higher resolutions. An initial validation of the solution procedure was performed by ensuring that, under several limiting conditions, known analytical or computational results were reproduced: (1) the Newman result for a finite-radius point electrode in the limit of a semi-infinite electrolyte ${ }^{20}$ (2) the conventional parallel plate resistor result in the limit of $r_{\mathrm{WE}}=r_{0}$; and (3) the spreading resistance result of Denhoff ${ }^{21}$ in the limit $r_{0} \rightarrow \infty$ and $k=\infty$.

An example of a typical computed potential distribution profile is shown in Fig. 1 with current distribution normal to the equipotential lines. Several points are noteworthy. First, as implied through the use of a global reaction constant, it is assumed here that the entirety of the interface between the working electrode and electrolyte is electrochemically active. That is, no attempt is made to simulate the real physical situation in which isolated triple-phase boundaries within this interface are the sites for electrochemical activity. Hence, the simulation yields equipotential lines that vary smoothly in the vicinity of the electrodes. Second, the majority of the flux follows the shortest path from the working electrode to the center of the counter electrode. That is, the current density is at a maximum at close to $r=0$ and falls rapidly as $r$ approaches $r_{0}$. Turning to the behavior of the potential, at the working electrode $\phi_{\mathrm{WE}}^{\text {electrolyte }}$ varies only slightly with radial position. In contrast, at the counter electrode, because of the concentration of the flux close to $r=0$, the potential $\phi_{\mathrm{CE}}^{\text {electrolyte }}$ displays a strong dependence on radial position. Indeed, at any given radial position, the ratio between interfacial current density and overpotential is simply the rate constant, as expressed in eqn (5), behavior that is captured in Fig. 1(c).

Given that the electrode overpotentials are not single-value quantities, it is not immediately obvious how one can compare the relative contributions of these overpotentials to the total voltage drop across the cell. Specifically, the challenge is to define a single-valued effective voltage at the electrolyte side of the interface. The approach taken here is as follows. It is proposed that the interfacial power dissipation, $P$, obtained 
from an integration of the position dependent quantities must equal the power dissipation obtained from the evaluation of single-valued parameters. Specifically, for the case of the counter electrode positioned at $y=0$, the power computed from a point-wise integration is

$$
P=2 \pi \int_{0}^{r_{0}} j(r, y=0) \cdot \phi(r, y=0) r \mathrm{~d} r
$$

whereas that expected from single-value parameters is

$$
P=I \eta_{\mathrm{CE}}^{\mathrm{eff}}=\left(2 \pi \int_{0}^{r_{0}} j(r, y=0) \cdot r \mathrm{~d} r\right) \eta_{\mathrm{CE}}^{\mathrm{eff}}
$$

where $I$ is the total current (easily computed as a single value), and $\eta_{\mathrm{CE}}^{\mathrm{eff}}$ is the effective electrode overpotential. Equating these two definitions of power yields

$$
\eta_{\mathrm{CE}}^{\mathrm{eff}}=\frac{\int_{0}^{r_{0}} j(r, y=0) \cdot \phi(r, y=0) r \mathrm{~d} r}{\left(\int_{0}^{r_{0}} j(r, y=0) \cdot r \mathrm{~d} r\right)}
$$

A similar expression is readily obtained for the working electrode by performing the integration at $y=t$. With these overpotentials so defined, the extent of isolation, $\xi$, is simply defined as the ratio of the overpotential at the working electrode, $\eta_{\mathrm{WE}}$, to the total electrode overpotential:

$$
\xi=\frac{\eta_{\mathrm{WE}}}{\eta_{\mathrm{WE}}+\eta_{\mathrm{CE}}}
$$

The closer this value to 1.0, the more effective the asymmetric geometry in providing the desired electrochemical data. For a geometry in which $r_{\mathrm{WE}} \leq r_{\mathrm{CE}}=r_{0}$ (with $k_{\mathrm{CE}}=k_{\mathrm{WE}}$ ) the isolation has a minimum possible value of 0.5 .

From the overpotential at the counter electrode, eqn (9) and the total current, the total interfacial resistance at the counter electrode can be computed:

$$
R_{\mathrm{CE}}=\frac{\eta_{\mathrm{CE}}}{I}
$$

If it is assumed that the area-specific interfacial resistance, $\tilde{R}$, and in particular that of the counter electrode, $\tilde{R}_{\mathrm{CE}}$, is a fixed material constant (equal to the inverse of the reaction rate constant, $k$ ), the total interfacial resistance then implies an effective area, and hence effective radius, $r_{\mathrm{CE}}^{\mathrm{eff}}$, over which the current density at the counter electrode is significant. Specifically,

$$
\tilde{R}_{\mathrm{CE}}=\frac{1}{k}=R_{\mathrm{CE}} \pi\left(r_{\mathrm{CE}}^{\mathrm{eff}}\right)^{2} \Rightarrow r_{\mathrm{CE}}^{\mathrm{eff}}=\sqrt{\frac{1}{\pi k R_{\mathrm{CE}}}}
$$

This radius value is indicated in Fig. 1(c) and it is evident that at $r=r_{\mathrm{CE}}^{\mathrm{eff}}$ the overpotential and current density have fallen significantly, to $9.5 \%$ of their respective peak values.

\section{Computational results}

While it is possible to evaluate the effective radius of the counter electrode, $r_{\mathrm{CE}}^{\mathrm{eff}}$, using the numerical procedures described above for any given set of input parameters, or even
Table 1 Range of numerical fit input parameters utilized in generating Fig. 2 and the parametric fit expressed in eqn (13). Computations carried out with $r_{0}(=3 \mathrm{~cm}) \gg r_{\mathrm{CE}}^{\text {eff }}$.

\begin{tabular}{llll}
\hline Parameter & Minimum & Maximum & Units \\
\hline Reaction rate constant, $k$ & 0.005 & 10 & $\left(\Omega \mathrm{cm}^{2}\right)^{-1}$ \\
Thickness, $t$ (with $\left.t>r_{\mathrm{WE}}\right)$ & 0.01 & 1.0 & $\mathrm{~cm}$ \\
Working electrode radius, $r_{\mathrm{WE}}$ & 0.001 & 0.2 & $\mathrm{~cm}$ \\
Conductivity, $\sigma$ & $1 \times 10^{-5}$ & 1 & $(\Omega \mathrm{cm})^{-1}$ \\
\hline
\end{tabular}

to evaluate the dependence of $r_{\mathrm{CE}}^{\mathrm{eff}}$ on a particular parameter with all others fixed, of greatest utility is a high-quality analytical approximation that enables immediate evaluation of $r_{\mathrm{CE}}^{\mathrm{eff}}$ (and $\xi$ ) without recourse to such a computation. The first step in establishing such an approximation is the selection of the appropriate parameter space of relevance. It can be readily anticipated that $r_{\mathrm{CE}}^{\mathrm{eff}}$ will depend on the material parameters, $\sigma$ and $k$, and on the geometric parameter $t$. In contrast, the geometric parameter $r_{\mathrm{WE}}$ can be expected to have negligible impact on the current and field lines when $r_{\mathrm{WE}}<t$, and, similarly, that $r_{0}$ will have no impact in the limit $r_{0} \rightarrow \infty$, expectations that are confirmed below. Accordingly, simulations were performed over a range of values of $\sigma, k, t$, $r_{\mathrm{WE}}$, as specified in Table 1, under the constraint that $r_{\mathrm{WE}}<t$ and for a fixed, large value of cell radius, $r_{0}$ (set to $3 \mathrm{~cm}$ ). Each parameter was varied over at least two orders of magnitude. Although the ranges for the material and geometric parameters are selected to represent typical experimental systems (see the Experimental section), no generality is lost as a consequence of the specific ranges selected. Each computation was composed of approximately 15000 grid points, whose spacing was refined to yield higher resolution in the vicinity of the electrode-electrolyte interfaces, as already noted above. Using 470 computed values of $r_{\mathrm{CE}}^{\mathrm{eff}}$ which obeyed the additional

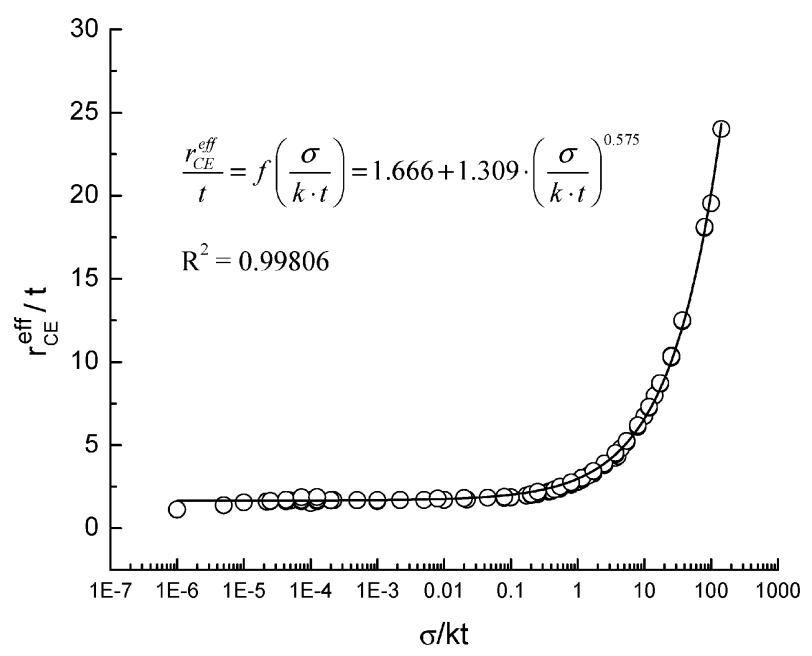

Fig. 2 Correlation between the dimensionless parameters $r_{\mathrm{CE}}^{\mathrm{eff}} / t$ and $\sigma / k t$, where $t$ is the electrolyte thickness, $\sigma$ is the electrolyte conductivity, $k$ is the electrode reaction rate constant, and $r_{\mathrm{CE}}^{\mathrm{eff}}$ is the computed, effective counter-electrode radius. Points are the results of the numerical calculations, limited to results for which $r_{\mathrm{CE}}^{\mathrm{eff}}<0.85 r_{0}$, and the line is the best fit to a phenomelogical, allometric function, with the fit equation as given. Range of parameters utilized for the generation of this figure are summarized in Table 1. 
constraint $r_{\mathrm{CE}}^{\mathrm{eff}}<0.85 r_{0}$, it was ultimately found that the results, Fig. 2, could be described by the allometric function:

$$
\begin{aligned}
& \frac{r_{\mathrm{CE}}^{\mathrm{eff}}}{t}=f\left(\frac{\sigma}{k t}\right)=1.666+1.309\left(\frac{\sigma}{k t}\right)^{0.575} \\
& \left(R^{2}=0.998\right)
\end{aligned}
$$

for $\sigma / k t$ values between $10^{-6}$ and 100 . As anticipated above, by restricting the geometries to those in which the radius of the working electrode is less than the electrolyte thickness, $r_{\mathrm{CE}}^{\mathrm{eff}}$ is independent of $r_{\mathrm{WE}}$.

Embodied in the results presented in Fig. 2 and the expression above is the dependence of $\xi$ on the material properties $\sigma$ and $k$ and geometric parameters $t$ and $r_{\mathrm{WE}}$. The explicit relationship is obtained by noting that the total current passing through each of the electrodes is the same, and furthermore that the reaction rate constants for the two electrodes are the same. Using these equalities and making the reasonable assumption that $r_{\mathrm{WE}}^{\mathrm{eff}} \approx r_{\mathrm{WE}}$, one obtains from (9)

$$
\xi=\frac{R_{\mathrm{WE}}}{R_{\mathrm{WE}}+R_{\mathrm{CE}}}==\frac{1}{1+\left(r_{\mathrm{WE}} / r_{\mathrm{CE}}^{\mathrm{eff}}\right)^{2}}
$$

where $r_{\mathrm{CE}}^{\mathrm{eff}}$ can be directly computed from (13).

As indicated above, it is to be expected that the solution to the Laplace equation with the given boundary conditions will be independent of cell radius in the limit $r_{0} \rightarrow \infty$. To identify the conditions under which $r_{0}$ becomes significant, a series of calculations were performed in which, for a fixed value of $\sigma / k$ $\left(=3.41 \times 10^{-2} \mathrm{~cm}\right)$ the overall cell radius was varied down to a small value $(0.1 \mathrm{~cm})$. In Fig. 3 the isolation is plotted as a function of overall cell radius for several specific values of the working electrode radius (in particular, $r_{\mathrm{WE}}=0.2 \mathrm{~mm}$, $0.4 \mathrm{~mm}$, and $0.691 \mathrm{~mm}$ ) for a cell with thickness $t=0.1 \mathrm{~cm}$. Beyond the expected observation that greater isolation is achieved for working electrodes with smaller contact areas, it is apparent that in all three configurations, the isolation is

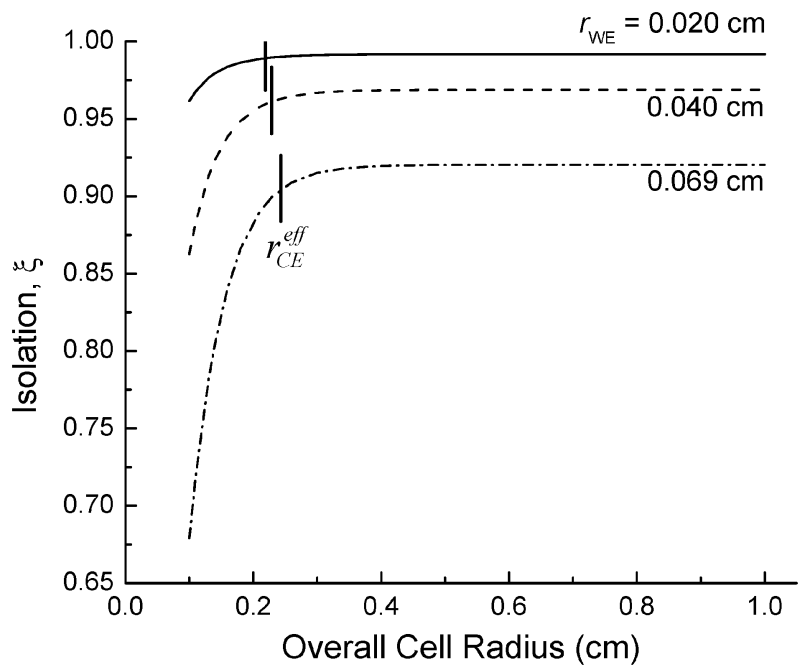

Fig. 3 Dependence of isolation, $\xi$, on overall cell radius, $r_{0}$, for 3 different working electrode radii (with values as indicated). Also shown are the effective counter electrode radii calculated for each of these three geometries from eqn (13), which applies when $r_{0}$ is large. Calculations performed for $k=0.615 \Omega^{-1} \mathrm{~cm}^{-2}, \sigma=0.021 \Omega^{-1} \mathrm{~cm}^{-1}, t=0.1 \mathrm{~cm}$. independent of $r_{0}$ once the overall cell radius reaches a value approximately $20 \%$ greater than $r_{\mathrm{CE}}^{\mathrm{eff}}$, consistent with the criterion used to generate the data set of Fig. 2 (the value of $r_{\mathrm{CE}}^{\mathrm{eff}}$ for each of the configurations is indicated directly on each plot). This behavior is consistent with the observation that the majority of the current flows through the central region of the cell and thus increasing the cell radius significantly beyond the calculated effective counter electrode radius has minimal impact on the potential profile. A key implication of this result is that for cells of sufficient radius, imprecision in placement of the working electrode does not impact the measured overpotential at that electrode. Such geometric insensitivity sharply contrasts that of typical reference electrode geometries in which slight misalignments in the placement of electrodes can have a major impact on the measured results, even in the d.c. limit explicitly considered here. ${ }^{1,2}$ By relaxing the requirements of precision placement of electrodes, the reference-less configuration greatly simplifies the experimental fabrication steps.

It is of some value to consider the physical origin of the dependence of isolation on the dimensionless quantity $\sigma / k t$. Referring to eqn (6), it is apparent that the overpotential $\eta$ is proportional to the local component of the gradient in the potential field along the direction normal to the interface, with the proportionality constant being the ratio of the two material parameters, $\sigma / k$. As a consequence, solutions for $\eta$, and accordingly, the isolation, depend not on the individual values of $\sigma$ and $k$ but rather on the ratio $\sigma / k$. The manner in which $\xi$ depends on this ratio is shown in Fig. 4 for several specific values of cell thickness, $t$, with a fixed working electrode radius, $r_{\mathrm{WE}}=0.1 \mathrm{~cm}$. Because $\sigma$ and $k$ were varied independently to obtain these data, it is possible to confirm that the resulting value of $\xi$ depends only on the ratio, as anticipated. Furthermore, the figure reveals that at a given $\sigma / k$ value, the isolation increases monotonically with thickness. This can be understood to result from the broadening of the current distribution at the counter electrode, which lowers the overpotential at that interface. Moreover, for a given cell thickness, it is apparent that the isolation reaches limiting

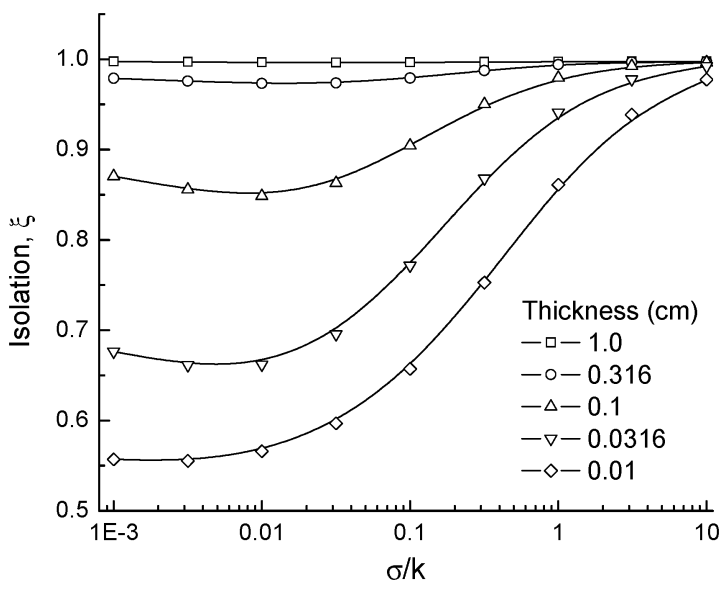

Fig. 4 Dependence of electrode isolation on the ratio of intrinsic material properties, $\sigma / k$, for varying cell thicknesses (as indicated), $r_{\mathrm{WE}}=0.1 \mathrm{~cm}$, and $r_{0}(=3 \mathrm{~cm}) \gg r_{\mathrm{CE}}^{\mathrm{eff}}$. Points represent computed values and solid lines are guides to the eye. 
values at both extrema in $\sigma / k$. At high $\sigma / k$ values, the electrolyte approaches a state of 'perfect' conductivity, and the isolation therefore approaches a value simply determined by the radii of the two electrodes. At low $\sigma / k$ values, the potential distribution within the electrolyte approaches that expected for perfect (reversible) electrodes, and the isolation approaches a value determined by $r_{\mathrm{WE}}$ and $t$. Indeed, as evident from eqn (13), in this limit, $r_{\mathrm{CE}}^{\mathrm{eff}}$ is simply $1.667 t$, and hence the isolation, via eqn (14), depends only on the ratio $r_{\mathrm{WE}} / t$. Finally, although the origin of the shallow minimum in $\xi$ at approximately $\sigma / k=0.1$ cannot be readily explained, this feature was confirmed (via extensive computations in this regime) to reflect the real behavior of the system, rather than a computational artifact of mesh resolution or convergence criterion.

\section{Experimental parameters}

Beyond cell geometry, two key experimental inputs are required for the evaluation of the potential profile and the extent of isolation of the voltage drop at the working electrode. These are the electrolyte conductivity, $\sigma$, and the reaction rate constant, $k$. These two terms were determined from an impedance measurement of an electrochemical cell with a simple, symmetric geometry. The $\mathrm{CsH}_{2} \mathrm{PO}_{4}$ electrolyte powder was synthesized from a stoichiometric aqueous solution of $\mathrm{Cs}_{2} \mathrm{CO}_{3}$ and $\mathrm{H}_{3} \mathrm{PO}_{4}$ as detailed elsewhere. ${ }^{22}$ Composite electrodes were prepared from platinum black (Sigma Aldrich-nom. $\sim 30 \mathrm{~m}^{2} / \mathrm{g}$ ) and $\mathrm{CsH}_{2} \mathrm{PO}_{4}$ combined in a 3:7 weight ratio and mechanically mixed. The tri-layer cell was fabricated by a co-pressing method in which the three powder layers were uniaxially cold-pressed between two $3 / 4$ " diameter porous stainless steel disks at $125 \mathrm{MPa}$ for $20 \mathrm{~min}$.

A.c. impedance measurements were carried out in an in-house constructed apparatus in which the sample is maintained at $240{ }^{\circ} \mathrm{C}$ under humidified conditions (to suppress dehydration $^{8}$ ) in a convection oven. The reactant gas, pure hydrogen, was supplied at a rate of $30 \mathrm{sccm}$ (MKS PR4000 mass flow control unit) and humidified by bubbling through water held at $78{ }^{\circ} \mathrm{C}\left(\mathrm{pH}_{2} \mathrm{O}(\mathrm{eq})=0.43 \mathrm{~atm}\right)$. The total gas space velocity at the cell was $1.4 \mathrm{~cm} / \mathrm{s}$. Data were collected in a four-wire configuration using an Eco Chemie Autolab PGSTAT302 equipped with a frequency response analysis module. Measurements were performed at a voltage amplitude of $10 \mathrm{mV}$ over frequencies ranging from $10 \mathrm{mHz}$ to $1 \mathrm{MHz}$.

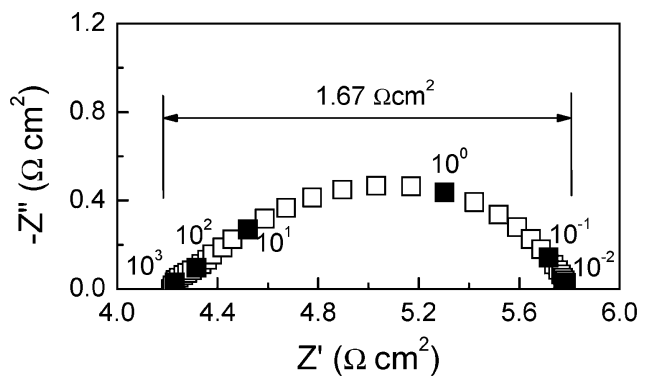

Fig. 5 Impedance spectrum collected from a Pt $+\mathrm{CsH}_{2} \mathrm{PO}_{4}$ $\left|\mathrm{CsH}_{2} \mathrm{PO}_{4}\right| \mathrm{Pt}+\mathrm{CsH}_{2} \mathrm{PO}_{4}$ symmetric cell at $240{ }^{\circ} \mathrm{C}$ under humidified hydrogen, with $p \mathrm{H}_{2} \mathrm{O}=0.43 \mathrm{~atm}$.
The impedance spectrum obtained from the symmetrical cell is shown in Fig. 5 in Nyquist form $\left(-Z_{\text {imag }}\right.$ vs. $Z_{\text {real }}$ as parametric functions of frequency). Because of the high conductivity of the electrolyte, the arc normally associated with the electrolyte response is not accessible. What is evident is the electrode arc which is displaced along the real axis from the origin, where the offset corresponds to the magnitude of the electrolyte resistance. While the electrode response appears nominally as a single arc in the Nyquist representation, it was found that the response could be best fit assuming two processes using an $\left(R_{\text {electrolyte }}\right)(R Q)(R Q)$ equivalent circuit (where $Q$ is a constant phase element with impedance $\left.Z_{Q}=A(i \omega)^{-\alpha}\right)$. The sum of the two resistances is used to represent the total interfacial impedance, and from this $k$ is simply (cell area $\times$ total interfacial resistance $)^{-1}$. The value measured here, $0.615 \Omega^{-1} \mathrm{~cm}^{-2}$, is roughly 30 times smaller than that implied from previous measurements of symmetric $\mathrm{CsH}_{2} \mathrm{PO}_{4}$-based cells with platinum-based electrode composites, ${ }^{22}$ with the difference resulting from differences in electrode preparation methods and overall composition. In contrast, the value of the electrolyte conductivity ( $\sigma=0.0210 \Omega^{-1} \mathrm{~cm}^{-1}$ ), obtained from the impedance at the high frequency limit, is in excellent agreement with earlier reported values. $^{22-25}$

\section{Experiments using the reference-less geometry}

For the experimental implementation of the reference-less method, two asymmetric cells of substantially different

dimensions were fabricated. Both cells employed a $\mathrm{CsH}_{2} \mathrm{PO}_{4}+\mathrm{Pt}$ composite mixture identical to that described above for both the counter and working electrodes. The area of the working electrode was limited by using a simple masking technique. After completion of the electrochemical measurements, the working electrode areas were measured by optical microscopy. The relevant dimensions for the two cells are as follows: cell $1, t=0.12 \mathrm{~cm}, r_{\mathrm{WE}}=0.684 \mathrm{~mm}$; cell 2 , $t=0.71 \mathrm{~cm}, r_{\mathrm{WE}}=0.348 \mathrm{~mm}$. The overall radius, $r_{0}$, for both cells was $0.953 \mathrm{~cm}$. Based on these dimensions, the measured material properties, and eqn (13), the predicted $r_{\mathrm{CE}}^{\mathrm{eff}}$ for the two cells are $0.276 \mathrm{~mm}$ and $1.346 \mathrm{~mm}$, respectively, implying respective isolation values of 0.942 and 0.997 . This explicit calculation reveals that greater isolation is generally obtained for greater thicknesses (for $\sigma / k t>1$, Fig. 2), but this isolation is achieved at the expense of higher system impedance, which can lead to greater noise in the experimental data.

Electrochemical impedance and polarization measurements were carried out using the same apparatus employed for symmetric cell characterization and under the same experimental conditions $\left(T=240{ }^{\circ} \mathrm{C}\right.$, atmosphere $=$ flowing, humidified hydrogen, with $\mathrm{pH}_{2} \mathrm{O}=0.43$ atm, gas-phase space-velocity $=1.4 \mathrm{~cm} / \mathrm{s})$. As above, the a.c. impedance measurements were performed with a $10 \mathrm{mV}$ amplitude around equilibrium at frequencies from $10 \mathrm{mHz}$ to $1 \mathrm{MHz}$. The d.c. polarization curves were collected under potentiostatic mode at a scan rate of $5 \mathrm{mV} / \mathrm{s}$. The voltage ranges were $\pm 300 \mathrm{mV}$ for the thinner cell and $\pm 400 \mathrm{mV}$ for the thicker cell. The latter was measured with a larger voltage excursion because the overall resistance is significantly higher. 


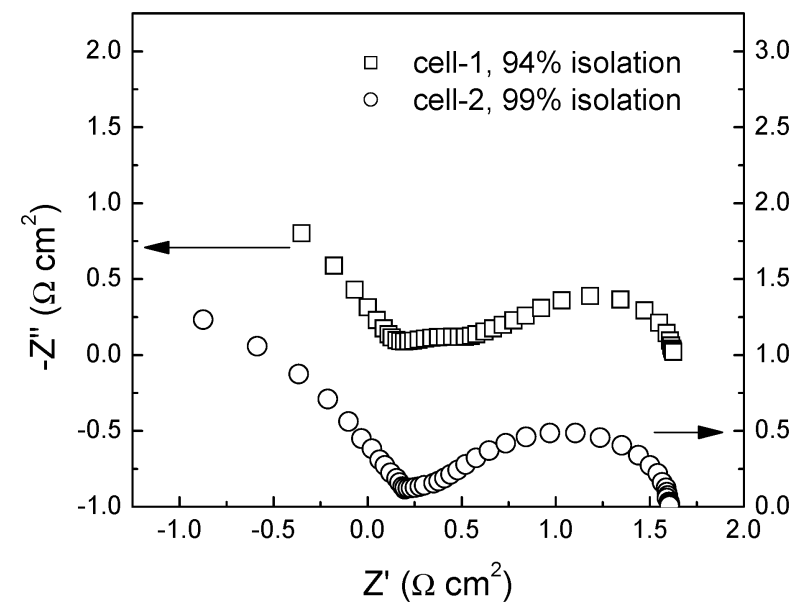

Fig. 6 Impedance spectra collected from $\mathrm{Pt}+\mathrm{CsH}_{2} \mathrm{PO}_{4}\left|\mathrm{CsH}_{2} \mathrm{PO}_{4}\right| \mathrm{Pt}$ $+\mathrm{CsH}_{2} \mathrm{PO}_{4}$ microelectrode cells (asymmetric geometry) at $240{ }^{\circ} \mathrm{C}$ under humidified hydrogen, with $p \mathrm{H}_{2} \mathrm{O}=0.43$ atm. Data are corrected for ohmic resistance of the electrolyte and normalized to the area of the working electrode.

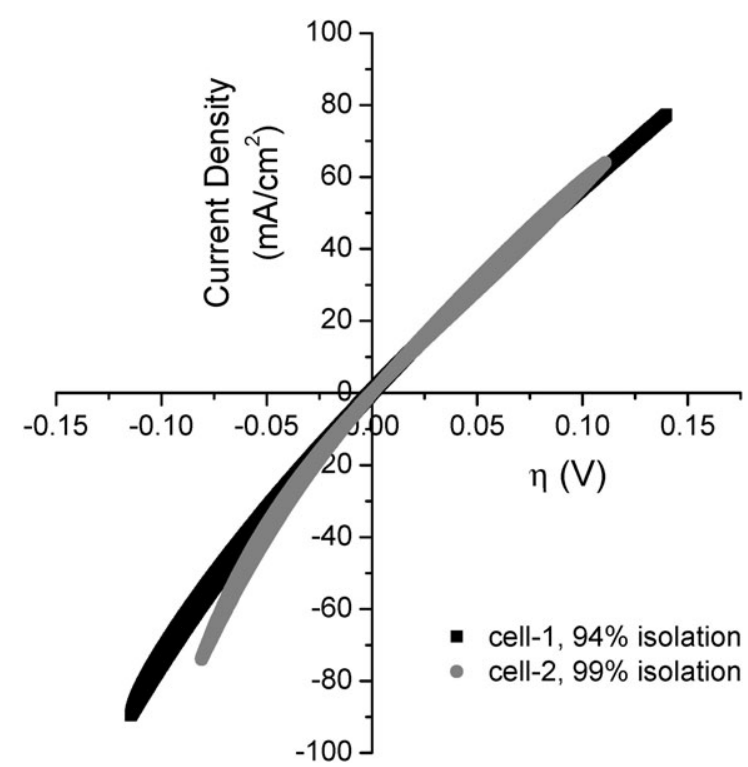

Fig. 7 IR-corrected polarization curves collected from $\mathrm{Pt}+$ $\mathrm{CsH}_{2} \mathrm{PO}_{4}\left|\mathrm{CsH}_{2} \mathrm{PO}_{4}\right| \mathrm{Pt}+\mathrm{CsH}_{2} \mathrm{PO}_{4}$ microelectrode cells (asymmetric geometry) at $240{ }^{\circ} \mathrm{C}$ under humidified hydrogen, with $p \mathrm{H}_{2} \mathrm{O}=0.43 \mathrm{~atm}$. Data are corrected for ohmic resistance of the electrolyte and normalized to the area of the working electrode.

\section{Results and discussion}

The measured impedance spectra and electrode polarization curves, for both cells, are presented in Fig. 6 and 7, respectively. From the impedance data the resistances due to the electrolyte and the electrodes (sum of the counter and working electrodes) are immediately available, and in both figures the results are corrected for the measured electrolyte resistance for ease of comparison. Implicit in this correction procedure is the assumption that the potential distribution within the electrolyte is independent of frequency, an assumption that is not explicitly examined here and indeed is often violated in configurations that include a reference electrode. ${ }^{2}$ As shown below, however, good agreement between measured and computed impedances is obtained, suggesting that such effects are negligible in the reference-less geometry.

Computationally, using the definition established for the electrode overpotentials, eqn (9), the voltage drop across the electrolyte is readily obtained and hence so is the electrolyte contribution to the system resistance, in addition to the resistances associated with each individual electrode. From the value of the potential drop across the electrolyte, it is noted that the strength of the electric field within the electrolyte remains below $3 \mathrm{~V} / \mathrm{cm}$, justifying the implicit assumption made in obtaining the electrode overpotential curves (Fig. 7) that the electrolyte is ohmic over the voltage range of the experiment.

In Table 2 a comparison is provided between the resistance values obtained from the complete calculation, the analytical prediction, and the experimental measurement in the reference-less geometry. The predicted value in the case of the counter electrode is that computed according to $\left(R_{\mathrm{CE}}=1 /\left(\pi\left(r_{\mathrm{CE}}^{\mathrm{eff}}\right)^{2} k\right)\right)$, where the allometric expression (13) to is used to obtain $r_{\mathrm{CE}}^{\text {eff }}$. In the case of the working electrode, it is simply the result directly implied by the electrode area $\left(R_{\mathrm{WE}}=1 /\left(\pi\left(r_{\mathrm{WE}}\right)^{2} k\right)\right)$. In the case of the 'experimentally' obtained isolation value, here again the allometric expression is used to obtain $r_{\mathrm{CE}}^{\mathrm{eff}}$ and, thereby, the determination of isolation is made independently of a direct physical measurement of $r_{\mathrm{WE}}$. That is, so long as the electrolyte thickness is greater than the radius of the working electrode, the computation yields $r_{\mathrm{CE}}^{\text {eff }}$ and the measurement yields the total electrode overpotentation, from which the isolation and $r_{\mathrm{WE}}$ can be deduced.

Overall, it is apparent that there is excellent agreement between the three sets of values presented in Table 2. The agreement between the computed and predicted electrode resistances (within a fraction of a percent for cell 1 , and within $3 \%$ for cell 2), as well as between the isolation values, indicates that the allometric function provides a satisfactory representation of the computational results. The agreement between the computed and experimental results, in turn, validates the computational approach.

The $I R$-corrected polarization curves reveal important aspects of the electrochemical reactions. At zero to positive bias, conditions that largely probe the hydrogen oxidation reaction (forward direction for eqn (1)), the two electrode polarization curves show excellent agreement and are extremely linear. At the origin, the slopes are 0.621 and $0.646 \Omega^{-1} \mathrm{~cm}^{-2}$ for cells 1 and 2 , respectively. These values are furthermore almost identical to the reaction rate constant obtained from the symmetric cell measurements $\left(0.615 \Omega^{-1} \mathrm{~cm}^{-2}\right)$ and confirm that a high degree of isolation has been achieved. That is, at zero (and positive) bias only the working electrode contributes to the measured interfacial impedance. With increasingly positive bias, the slopes decrease very slightly to values of 0.519 and $0.556 \Omega^{-1} \mathrm{~cm}^{-2}$, respectively, for the two cells. At negative bias, at which the proton reduction reaction is probed, the polarization curves are detectably non-linear 
Table 2 Comparison between computed, predicted and measured cell resistances for cells of two differing geometries and hence expected isolation values. Cell-1: $t=0.12 \mathrm{~cm}, r_{\mathrm{WE}}=684 \mu \mathrm{m}, r_{0}=0.953 \mathrm{~cm}, r_{\mathrm{CE}}^{\text {eff }}=0.276 \mathrm{~cm} ;$ Cell-2: $t=0.71 \mathrm{~cm}, r_{\mathrm{WE}}=348 \mu \mathrm{m}, r_{0}=0.95 .3 \mathrm{~cm}, r_{\mathrm{CE}}^{\text {eff }}=0.135 \mathrm{~cm}$.

\begin{tabular}{|c|c|c|c|c|c|c|}
\hline \multirow[b]{2}{*}{ Resistance $(\Omega)$} & \multicolumn{3}{|l|}{ Cell-1 } & \multicolumn{3}{|l|}{ Cell-2 } \\
\hline & Computed $^{a}$ & Predicted & Measured & Computed $^{a}$ & Predicted & Measured \\
\hline Electrolyte & 141.5 & $\mathrm{n} / \mathrm{a}$ & 142.1 & 626.0 & $\mathrm{n} / \mathrm{a}$ & 659.3 \\
\hline WE & 110.7 & $110.6^{b}$ & $\mathrm{n} / \mathrm{a}$ & 438.0 & $427.9^{b}$ & $\mathrm{n} / \mathrm{a}$ \\
\hline $\mathrm{CE}$ & 7.3 & $6.8^{c}$ & $\mathrm{n} / \mathrm{a}$ & 0.6 & $0.3^{c}$ & $\mathrm{n} / \mathrm{a}$ \\
\hline Total electrode & 118.0 & $117.3^{d}$ & 115.5 & 438.6 & 428.2 & 425.2 \\
\hline Isolation $(\%)$ & 93.8 & $94.3^{d}$ & $94.1^{e}$ & 99.9 & $99.9^{d}$ & $99.9^{e}$ \\
\hline
\end{tabular}

${ }^{a}$ Directly computed using the definition of overpotential provided in eqn (8); ${ }^{b}$ Estimated based on the area of the working electrode and the assumption of a constant current density across the interface between the working electrode and the electrolyte; ${ }^{c}$ Predicted on the basis of eqn (13) (analytical expression); ${ }^{d}$ Predicted based on comments (b) and (c); ${ }^{e}$ Determined based on comment (c), from which the counter-electrode resistance is evaluated, and from the experimentally measured value for the total electrode resistance.

and diverge, with the slope for cell 1 reaching $1.1 \Omega^{-1} \mathrm{~cm}^{-2}$ (at $\eta=-0.12 \mathrm{~V}$ ) and that for cell 2 reaching $1.4 \Omega^{-1} \mathrm{~cm}^{-2}$ (at $\eta=-0.7 \mathrm{~V}$ ). These two effects (non-linearity and divergence) are, in fact, manifestations of the same phenomenon. As proton oxidation occurs at the working electrode, hydrogen reduction, with its smaller reaction rate constant, occurs at the counter electrode. With increasing forward bias, the relative contribution of the working electrode to the sum of the electrode overpotentials decreases, and the extent of isolation decreases relative to the value at zero bias. Accordingly, the data become more and more 'polluted' with the contribution of the counter electrode and this effect is greater for the cell with a lower initial isolation value. This result indicates that an extremely high degree of isolation at zero bias is required if the experiment is to retain a sufficiently high level of isolation over the entire voltage range of interest to permit, for example, a detailed mechanistic analysis.

Considering only the results at zero bias, in the case of cell 1 , the $94 \%$ isolation indicates that attribution of all of the non-electrolyte impedance to the working electrode leads to an overestimation of the potential drop at this electrode by $\sim 5 \%$. Consequently, an assumption without validation that the electrode overpotential is sufficiently isolated at the working electrode for an apparently attractive geometry can easily lead to an underestimation of the electrochemical activity of an electrode material. Alternatively, if one follows the standard approach of using electrolyte resistance to calculate electrode area from Newman's equation, and then again makes the assumption that the counter electrode resistance is negligible, one would overestimate the radius of the working electrode by $23 \%$. With this radius, the calculated total electrode resistance would be underestimated by $37 \%$. These large under and over estimates demonstrate the importance of accurately assessing the level of true isolation in a micro- or point electrode geometry.

These cautionary points notwithstanding, it is apparent that even cell 1, with a zero-bias isolation value of 0.94 , has a sufficiently confined working electrode such that the asymmetry of the electrochemical reaction, eqn (1), is revealed. It is noteworthy that the hydrogen oxidation reaction, the direction that is relevant for fuel cell applications, remains linear over the range of overpotentials that are typically encountered at the anode. The implication is that the reaction rate constant obtained from a symmetric cell measurement is, in the case of $\mathrm{CsH}_{2} \mathrm{PO}_{4}$-based fuel cells with Pt-based electrodes, applicable for a phenomenological description of anode behavior. It is also quite apparent that the electrochemical reaction does not obey the Butler-Volmer equation, ${ }^{26}$ which predicts a reaction rate constant that increases with increasing (positive or negative) bias from zero. While a mechanistic treatment of the reaction pathway is left for future investigation, the observation of linear behavior may have relatively immediate importance for macroscale fuel cell modeling.

\section{Conclusions}

It is demonstrated that, as alluded to in the literature, a microelectrode geometry can be utilized to directly probe the electrochemical characteristics of a single electrode without the requirement of a reference electrode. The microelectrode geometry can constrain the electrode potential drops in the system such that the majority of the measured electrochemical impedance is due to the working electrode of interest. A direct correlation between isolation and geometric and material parameters is presented here. From the complete numerical computations, the relationship, specifically, the dependence of the effective area of the counter electrode on the dimensionless quantity $\sigma / k t$, is explicitly mapped to a simple functional form. From $r_{\mathrm{CE}}^{\mathrm{eff}}$ and the assumption that the potential at the small, working electrode does not vary with radial position within $r_{\mathrm{WE}}$, the extent of isolation is readily computed. It is further shown that geometries which might intuitively appear to provide sufficient isolation, can lead to gross over or under estimates of material properties. Isolation at the 95\% level leads directly to an overestimation of the impedance of the working electrode by $\sim 5 \%$. Alternatively, if the area of the working electrode is unknown, use of the Newman relationship to obtain this value from a measurement of the electrolyte resistance can lead to a very large overestimate, and a correspondingly large underestimate of the area specific impedance of this electrode. Furthermore, if the extent of isolation changes with bias, as would generally be expected because of the asymmetry of the electrochemical reactions, interpretations of the polarization curves become increasingly difficult. Thus, direct determination of the extent of isolation is critical for the accurate determination of electrochemical behavior. With sufficient isolation, the reference-less geometry has the distinct advantage, as shown computationally, of being 
largely insensitive to electrode placement. This stands in contrast to the strict requirements for accurate electrode placement in a conventional experiment utilizing a reference electrode.

The methodology developed here is applied to the study of hydrogen electro-oxidation at the $\mathrm{Pt}-\mathrm{CsH}_{2} \mathrm{PO}_{4}$ interface. The forward and reverse reactions (hydrogen oxidation and proton reduction, respectively) are measurably different, with hydrogen electro-oxidation displaying almost linear behavior and proton electro-reduction displaying greater non-linearity and greater impedance at any given current density. Overall, the reaction does not follow classical Butler-Volmer characteristics. The combined experimental and computational approach demonstrated here provides a means for carefully probing this reaction and establishing the reaction pathway.

\section{Acknowledgements}

Funding for this work has been provided by Superprotonic, Inc. and by the Moore Foundation through its support of the Caltech Center for Sustainable Energy Research. We are grateful for insightful discussions with Dr Calum Chisholm and Dr Strahinja (Zeke) Zecevic of Superprotonic, Inc., and Dr David Goodwin of Caltech.

\section{References}

1 J. Rutman and I. Riess, Electrochim. Acta, 2007, 52(20), 6073-6083.

2 S. B. Adler, B. T. Henderson, M. A. Wilson, D. M. Taylor and R. E. Richards, Solid State Ionics, 2000, 134(1-2), 35-42.

3 P. Fabry and M. Kleitz, J. Electroanal. Chem., 1974, 57(2), $165-177$.

4 J. Mizusaki, H. Tagawa, T. Saito, K. Kamitani, T. Yamamura, K. Hirano, S. Ehara, T. Takagi, T. Hikita, M. Ippommatsu, S. Nakagawa and K. Hashimoto, J. Electrochem. Soc., 1994, 141(8), 2129-2134.

5 A. Bieberle, L. P. Meier and L. J. Gauckler, J. Electrochem. Soc., 2001, 148(6), A646-A656.
6 A. Bieberle and L. J. Gauckler, Solid State Ionics, 2000, 135(1-4, Sp. Iss. SI), 337-345.

7 E. Koep, D. S. Mebane, R. Das, C. Compson and M. L. Liu, Electrochem. Solid State Lett., 2005, 8(11), A592-A595.

8 R. J. Aaberg, R. Tunold, M. Mogensen, R. W. Berg and R. Odegard, J. Electrochem. Soc., 1998, 145(7), 2244-2252.

9 N. Bonanos and M. Mogensen, Solid State Ionics, 1997, 97(1-4), 483-488.

10 J. Fleig, F. S. Baumann, V. Brichzin, H. R. Kim, J. Jamnik, G. Cristiani, H. U. Habermeier and J. Maier, Fuel Cells, 2006, 6(3-4), 284-292.

11 V. Brichzin, J. Fleig, H. U. Habermeier and J. Maier, Electrochem. Solid State Lett., 2000, 3(9), 403-406.

12 V. Brichzin, J. Fleig, H. U. Habermeier, G. Cristiani and J. Maier, Solid State Ionics, 2002, 152(Part A Sp. Iss. SI), 499-507.

13 F. S. Baumann, J. Fleig, M. Konuma, U. Starke, H. U. Habermeier and J. Maier, Solid Oxide Fuel Cells IX, 2005, PV 2005-07, 1636.

14 F. S. Baumann, J. Fleig, M. Konuma, U. Starke, H. U. Habermeier and J. Maier, J. Electrochem. Soc., 2005, 152(10), A2074-A2079.

15 K. A. Sasaki and S. M. Haile, Under preparation.

16 T. Uda, Y. Awakura, S. Katsura and Y. Taninouchi, Proceedings of the 58th Annual meeting of the International Society of Electrochemistry, Banff, Canada, September 2007.

17 D. A. Boysen, T. Uda, C. R. I. Chisholm and S. M. Haile, Science, 2004, 303, 68-70.

18 T. Uda and S. M. Haile, Electrochem. Solid State Lett., 2005, 8, A245-A246.

19 G. D. Smith, in Numerical Solution of Partial Differential Equations: Finite Difference Methods, Oxford University Press, 3rd edn, 1985, ch. 5, pp. 239-248.

20 J. Newman, J. Electrochem. Soc., 1966, 113, 501-502.

21 M. W. Denhoff, J. Phys. D: Appl. Phys., 2006, 39, 1761-1765.

22 S. M. Haile, C. R. I. Chisholm, K. A. Sasaki, D. A. Boysen and T. Uda, Faraday Discuss., 2007, 134, 17-39.

23 A. I. Baranov, V. P. Khiznichenko, V. A. Sandler and L. A. Shuvalov, Ferroelectrics, 1988, 81, 1147-1150.

24 J. Otomo, N. Minagawa, C. J. Wen, K. Eguchi and H. Takahashi, Solid State Ionics, 2003, 156, 357-369.

25 Y. Taninouchi, T. Uda, Y. Awakura, A. Ikeda and S. M. Haile, J. Mater. Chem., 2007, 17(30), 3182-3189.

26 A. J. Bard and L. R. Faulkner, in Electrochemical Methods: Fundamentals and Applications, John Wiley \& Sons, New Jersey, 2nd edn, 2001, ch. 3, p. 100. 